\title{
Cold quark matter in astrophysics of compact stars
}

\author{
Armen Sedrakian \\ Institute for Theoretical Physics, J. W. Goethe-University, D-60438 Frankfurt am Main, Germany
}

\begin{abstract}
The appearance of quark matter in the centers of compact stars has a number of astrophysical implications. In this contribution I discuss the structure and stability of compact stars, gravitational radiation from non-axisymmetric deformations, and nucleation and dynamics of vortices (flux tubes) in the color superconducting phases.
\end{abstract}

Keywords: Neutrons stars, Quark-gluon plasma in quantum chromodynamics, Vortex dynamics, Aharonov-Bohm effect, Transport processes in quantum fluids

PACS: 97.60.Jd, 12.38.Mh, 47.32.C-, 03.65.Ta, 67.10.Jn

\section{INTRODUCTION}

The deconfinement phase transition in dense matter, a manifestation of asymptotic freedom of quantum chromodynamics (QCD), may lead to stable quark matter cores in massive compact stars. The deconfined ("liberated") quarks occupy continuum of scattering states, which in the low-temperature and high-density regime fill in the modes in a Fermi sphere. The behavior of quark matter in this regime resembles that of less exotic low-temperature systems found in condensed matter (e.g. electron gas or ultracold atomic vapor) or hadronic physics (e.g. nuclear or neutron matter) [1]. In analogy to these system the attractive interaction between quarks, mediated by the gluon exchange, which is responsible for the bound state spectrum of QCD, leads to quark superconductivity and superfluidity. This phenomenon is commonly refered to as color superconductivity [2, 3]. Interestingly, the inhomogeneous superconducting phases originally proposed in condensed matter and nucleonic system find their natural realization in compact stars when the $\beta$-equilibrium condition is imposed [4].

In the following, I will review and summarize some recent results concerning the astrophysical implications of the hypothesis of deconfinement and color superconductivity in quark matter. This contribution is not intended to be a comprehensive review of the field and is centered mainly upon my recent research.

\section{EQUILIBRIUM AND STABILITY}

While we are confident that neutron stars, which are observed as isolated pulsars or $\mathrm{X}$-ray sources in binaries, contain nuclear matter in their interiors, the existence of hybrid stars with central cores made of quark matter is not obvious. Qualitatively, the uncertainty arises because deconfined quark matter has larger number of degrees of freedom (color) than the ordinary nuclear matter and, consequently, its formation leads 
to a softening of the equation of states. Such softening may be fatal to the gravitational stability of the whole system; in fact, in many models of quark matter equation of state no stable configurations are found - the stars collapse into a black hole. A less severe uncertainty arises from the unknown physics of deconfinement and the density at which it occurs. The central densities of massive neutron stars are at about five times the nuclear saturation density or more. The nucleons will hardly remain intact at such high densities and the most plausible outcome of compression is a quark-gluon-fluid.

As an illustration, I start with the study of ref. [5], where the problem of equilibrium and stability of non-rotating and rapidly rotating hybrid stars was addressed. This work describes the quark matter in the Nambu-Jona-Lasinio (NJL) model, which is a lowenergy, non-perturbative approximation to QCD, that is anchored in the low-energy phenomenology of the hadronic spectrum. While dynamical symmetry breaking, by which quarks acquire mass, is incorporated in this model, it lacks confinement. A procedure which overcomes this difficulty in an ad hoc manner is matching the quark equation of state to some low-density equation of state of hadronic matter. This can be done either via Gibbs or Maxwell construction; both constructions lead to quantitatively similar result, therefore we have adopted the latter. In that case, at the deconfinement phase transition there is a jump in the density at constant pressure as illustrated in Fig. 1, left panel. The low-density nuclear equations of state are based on the Dirac-Bruckner-HartreeFock approach $[6,7]$. The choice of a particular nuclear equation of state is dictated by the need to match it to the quark equation of state. It turns out that it should be rather hard in order to fulfill this requirement Alternatively, one may consider the density at which the deconfinement takes place as a free parameter by adding an effective bag constant to the quark matter equation of state. In these, more phenomenological approaches, softer nuclear equations of state can be accommodated [8]. If vector interactions are included in the NJL model Lagrangian the stability of the models is improved and softer nuclear equations of state can be accommodated as well [9].

The spherically symmetric solutions of Einstein's equations for self-gravitating fluids are given by the well-known Tolman-Oppenheimer-Volkoff equations [6, 7]. A generic feature of these solutions is the existence of a maximum mass for any equation of state; as the central density is increased beyond the value corresponding to the maximum mass, the stars become unstable towards collapse to a black hole. A criterion for the stability of a sequence of configurations is the requirement that the derivative $d M / d \rho_{c}$ should be positive (the mass should be an increasing function of the central density). At the point of instability the fundamental (pulsation) modes become unstable. If stability is regained at higher central densities, the modes by which the stars become unstable towards the eventual collapse belong to higher-order harmonics.

For configurations constructed from a purely nuclear equation of state the stable sequence extends up to a maximum mass of the order $2 M_{\odot}$ (Fig. 1, right panel), this large value being a consequence of hardness of the equation of state. The hybrid configurations branch off from the nuclear configurations when the central density reaches that of the deconfinement phase transition. It is seen that a stable branch of hybrid stars emerges in the range of central densities $1.3 \leq \rho_{c} \leq 2.5 \times 10^{15} \mathrm{~g} \mathrm{~cm}^{-3}$. The masses of the quark cores cover the range $0 \leq M_{\text {core }} / M_{\odot} \leq 0.75-0.88$ for central densities $1.3 \times 10^{15} \leq \rho_{c} \leq 2 \times 10^{15}$. Thus, the quark core mass ranges from one third to about the half of the total stellar mass. 

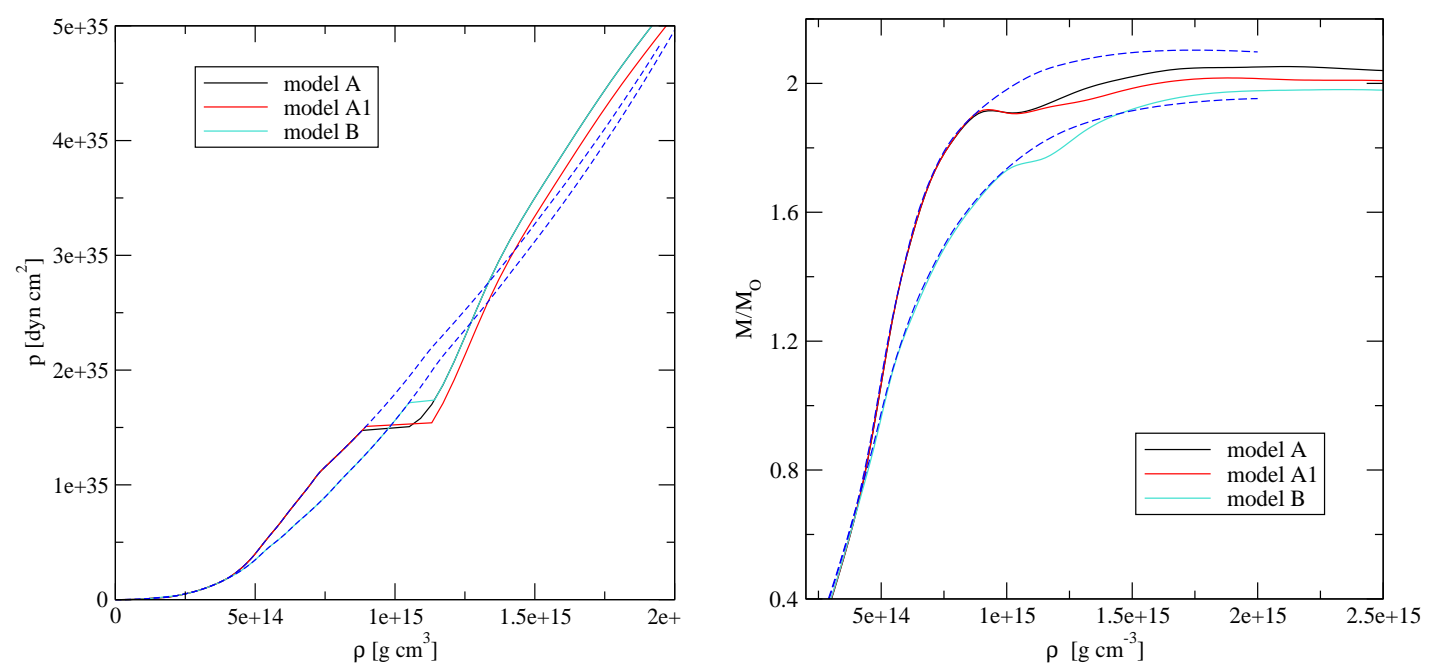

FIGURE 1. Left panel. Number density versus pressure for three models. For the models A and A1 the nuclear (low density) equation of state is the same; for the models A and B the quark (high density) equation of state is the same. Right panel. Dependence of the total stellar mass and the mass of the quark core in units of solar mass $M_{\odot}$ on the central density for non-rotating configurations. The lower set of curves represents the masses of the CCS quark cores, the upper set - the total masses of the configurations. The maximal masses are marked with boxes.

\section{Rapidly rotating stars}

Millisecond neutron stars can rotate at frequencies which are close to the limiting orbital Keplerian frequency at which mass shedding from the equatorial plane starts. The Keplerian frequency sets an upper limit on the rotation frequency, since other (less certain) mechanisms, such as secular instabilities, could impose lower limits on the rotation frequency. The mass versus central density dependence of compact stars rotating at the Keplerian frequency is similar to that for non-rotating stars with the scales for mass shifted to larger values [5]. The increase in the maximum mass for stable hybrid configurations (in solar mass units) is $2.052 \rightarrow 2.462$ for the model A, $2.017 \rightarrow 2.428$ and 2.4174 for the model A1 (there are two maxima) and $1.981 \rightarrow 2.35$ for the model B [5].

The recent discovery of the double-pulsar system PSR J0737-3039 [10, 11] offers a unique opportunity to place further bounds on the gross parameters of compact stars by a measurement of the moment of inertia of star $\mathrm{A}$, since the spin frequencies and the masses of both pulsars are accurately measured. Timing measurements over a period of years could provide information on spin-orbit coupling which could be revealed through an extra advancement of the periastron of the orbit above the standard post-Newtonian advance or in the precession of the orbital plane about the direction of the total angular momentum [12]. The dependence of the moment of inertia, $I$, of configurations on their mass (in the case of rotation at the Keplerian frequency) is shown in Fig. 2. Since the moment of inertia is independent of the rotation frequency, we have chosen to extract it for configurations rotating at the limiting frequency; note that the masses of configurations should be rescaled appropriately, if one is interested in the $I(M)$ function 

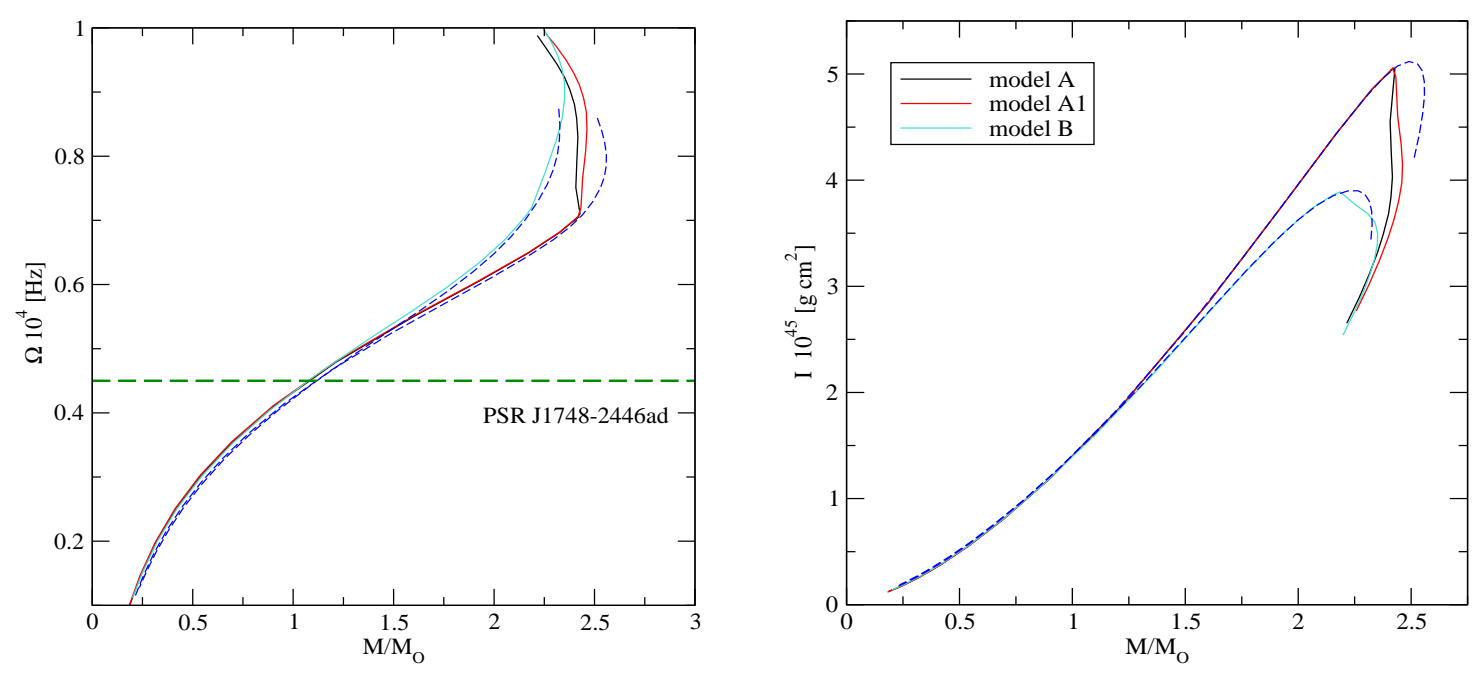

FIGURE 2. Left panel. Dependence of Keplerian (maximal) frequency of rotation for hybrid stars on their mass (in solar mass units). Right panel. Dependence of moment of inertia of the hybrid configurations on the mass of configuration (in solar mass units) rotating at the limiting frequency. The conventions are the same as in Fig. 1.

for slowly rotating configurations. It is seen that while an accurate measurement of the moment of inertia of pulsar A can discriminate between the two nuclear equations of state, it will not be useful for accessing the properties of hybrid stars since the measured masses of pulsars are too low: $M / M_{\odot}=1.337$ for pulsar A and $M / M_{\odot}=1.250$ for pulsar B; as apparent from Fig. 2 observations of heavier objects are needed to obtain useful bounds on the moment of inertia of hybrid configurations. The differences $\sim 20 \%$ in the moments of inertia of purely nuclear and hybrid configurations of the same mass (in the case of the models A and A1) are within the accuracy that can be achieved in measurements similar to those carried out for PSR J0737-3039.

\section{Gravitational radiation}

It is interesting that direct observational imprint of the size and mass distribution of the quark core will be carried by continuous gravitational waves if the core is crystallinesolid $[13,14,15]$ or superconducting-solid [16, 17, 18, 19]. Indeed, gravitational waves emitted by non-axisymmetric rotating compact stars are expected to be in the bandwidth of current gravitational wave interferometric detectors. Gravity waves arise from time-dependent quadrupole deformations of masses. In hybrid stars the axial symmetry of the star's core can be broken by the solid deformations in CCS matter. Several authors estimated the strength of gravitational wave emission from crystallinecolor-superconducting (CCS) matter [17, 18, 19]. The characteristic strain amplitude of gravitational waves emitted by a triaxial star rotating about its principal axis is $h_{0}=16 \pi^{2} G c^{-4} \varepsilon I_{z z} v^{2} r^{-1}$, where $v$ is the star's rotation frequency, $r$ is the distance to the observer, $\varepsilon=\left(I_{x x}-I_{y y}\right) / I_{z z}$ is the equatorial ellipticity, $I_{i j}$ is the tensor of moment of inertia, $G$ is the gravitational constant, $c$ is the speed of light. The elastic deforma- 
tions are assumed to be small perturbation on the background equilibrium of the star. The current upper limit for the Crab pulsar $h_{0}<3.4 \times 10^{-25}$, which is rotating at the frequency $v=29.6 \mathrm{~Hz}$ at the distance $2 \mathrm{kpc}$ [20]. The key unknown parameter of the theory is the breaking strain of CCS core $\bar{\sigma}_{\max } \sim 10^{-3}-10^{-5}$ and the shear modulus, which can be computed approximately and scales as $\mu_{\text {shear }} \sim \Delta^{2}$, where $\Delta$ is the pairing gap in CCS matter [16]. It follows then that $h_{0} \propto \bar{\sigma}_{\max } \Delta^{2}$. The strain of gravitational wave emission turns out to be close to the upper limit quoted above if $\Delta=50 \mathrm{MeV}$ and $\bar{\sigma}_{\max }=10^{-4}$. The Crab pulsar's current limit implies $\bar{\sigma}_{\max } \Delta^{2} \sim 0.25 \mathrm{MeV}^{2}$, assuming maximally strained matter. The evolutionary avenues that may lead to such maximal deformations are not known. Pulsars may simply preserve their initial deformations as they cool down and solidify. Additional contribution due to the deformations in the crust of the star have not been taken into account in our estimates. However, this contribution is likely to relevant to low-mass neutron stars [21].

\section{COLOR-MAGNETIC FLUX TUBES}

The information gained from measurements of the gross parameters of compact objects, such as the mass or the moment of inertia give us only indirect information on potential phases of matter in their interiors. The study of rotational, thermal and magnetic evolutions of neutron stars provide complementary information, which in many cases is much more sensitive to the composition of matter.

This section discusses the response of dense quark matter to magnetic field following Ref. [22]. Our discussion will be focused on the formation and dynamics of flux tubes in the two-flavor color superconducting (2SC) phase. The intact gauge symmetry in the 2SC phase is a linear combination of the ordinary electromagnetic and color symmetries; this phenomenon is called "rotated electromagnetism" [23]. The associated gauge field, the " $\tilde{Q}$ photon", is a combination of the original photon and one of the gluons. It is massless and propagates freely in 2SC quark matter. The orthogonal combination $X$ is a broken gauge generator, and the associated magnetic field has a finite penetration depth.

Depending on the ratio of the $X$-flux penetration depth to the coherence length of the condensate, the 2SC phase may be type-I or type-II with respect to the $X$ magnetic field $[24,25]$. A superconductor is of type II if it obeys the condition $\kappa \equiv \lambda / \xi>1 / \sqrt{2}$, where $\kappa$ is the Ginzburg-Landau (GL) parameter, $\lambda$ is the penetration depth, and $\xi$ is the coherence length of the superconductor. For typical values of $2 \mathrm{SC}$ superconductor $\kappa_{2 \mathrm{SC}} \approx 11 \Delta / \mu_{q}$. Therefore, 2SC quark matter will be of type II if the pairing gap is sufficiently large, $\Delta \geq \mu_{q} / 16$. In quark matter we expect $\mu_{q} \sim 400 \mathrm{MeV}$, so this only requires the $2 \mathrm{SC}$ pairing gap to be greater than about $25 \mathrm{MeV}$. Similar conclusions were reached in refs. [24, 25].

Type-II superconductivity leads to formation of flux tubes if the cost of inserting a flux tube, which has always a positive self-energy (flux-tension) in a superconductor is compensated by the interaction energy of the flux tube with external magnetic field intensity. The critical intensity (lower critical field) $H_{c 1}$ for the formation of Abrikosov flux tubes containing $X$-magnetic flux is large, of order $10^{17} \mathrm{G}$. However, we argued that, when the quark matter cools into the 2SC phase, the process of domain formation and amalgamation is likely to leave some of the $X$ flux trapped in the form of flux tubes. 
The exact configuration and density of such tubes depends on details of the dynamics of the phase transition, but the density could be within an order of magnitude of the density of conventional flux tubes in proton-superconducting nuclear matter. Note that the calculations apply to $2 \mathrm{SC}$ quark matter in the temperature range $T_{1 S C}<T \ll T_{2 S C}$ where $T_{2 S C}$ is the critical temperature for the formation of the $2 \mathrm{SC}$ condensate while $T_{1 S C}$ is the critical temperature of formation of spin one condensate.

We shall assume that the flux tubes in the 2SC phase are topologically stable. This is not obvious, since they are analogous to "Z-strings" of the standard model [26] which have been found to be stable only in a small region of the standard model parameter space [27], although the stable region may be enlarged when bound states are taken in to account [28]. Because there are also differences between the 2SC phase of QCD and the Higgs phase of the standard model, a separate stability calculation will be needed for the $2 \mathrm{SC}$ case.

The 2SC phase contains three species of gapless fermions: two quarks ("blue up" and "blue down") and the electron. These are expected to dominate its transport properties. Strange quarks can be included in the analysis as long as their pairing pattern does not break the $\tilde{Q}$ gauge symmetry. Muons may also be present, but, like strange quarks, their higher mass gives them a lower Fermi momentum so they make a sub-leading contribution to the transport phenomena.

Light ungapped fermions will be scattered from flux-tubes due to the Aharonov-Bohm effect; the cross-section of this process is given by [29]

$$
\frac{d \sigma}{d \vartheta}=\frac{\sin ^{2}(\pi \tilde{\beta})}{2 \pi k \sin ^{2}(\vartheta / 2)}
$$

where $\tilde{\beta}=q_{p} / q_{c}$, where $q_{p}$ is the charge of the scattering particle. For a flux tube that arises as a topological soliton in an Abelian Higgs model, $q_{c}$ is the charge of the condensate field whose winding by a phase of $2 \pi$ characterizes the flux tube; $k$ is the momentum in the plane perpendicular to the string, and $\vartheta$ is the scattering angle. Aharonov-Bohm scattering has several important features: (i) The cross-section vanishes if $\tilde{\beta}$ is an integer, but is otherwise non-zero. (ii) The cross section is independent of the thickness of the flux tube: the scattering is not suppressed in the limit where the symmetry breaking energy scale goes to infinity, and the flux tube thickness goes to zero. (iii) The cross section diverges both at low energy and for forward scattering.

The values of $\tilde{\beta}$ for scattering of the fermions that are ungapped in the 2SC phase off a flux tube containing magnetic flux is given, to lowest order in the electromagnetic coupling constant $\alpha$, by

$$
\tilde{\beta}^{\psi}=\operatorname{diag}\left(\frac{1}{2}+\frac{\alpha}{2 \alpha_{s}}, \frac{1}{2}-\frac{\alpha}{2 \alpha_{s}}, \frac{1}{2}-\frac{\alpha}{2 \alpha_{s}}, \frac{1}{2}+\frac{\alpha}{2 \alpha_{s}},-1+\frac{\alpha}{\alpha_{s}},-1,-\frac{\alpha}{\alpha_{s}}\right)
$$

in the basis defined as $\psi=\left(r u, g d, r d, g u, b u, b d, e^{-}\right)$, where " $r u$ " means the red up quark, etc. " $e$ " is the electron and $\alpha_{s} \sim 1$ is the strong coupling constant. We conclude that the gapped quarks have $\tilde{\beta}$ close to 0.5 , which means that they have near-maximal Aharonov-Bohm interactions with an $X$-flux tube. Among the lighter (and hence more phenomenologically relevant) fermions, the $\tilde{Q}$-neutral $b d$ has zero Aharonov-Bohm 
interaction with the flux tube, while the $b u$ and electron have the same AharonovBohm factor $\sin \left(\pi \tilde{\beta}^{b u}\right)=\sin \left(\pi \tilde{\beta}^{e}\right) \approx-\pi\left(\alpha / \alpha_{S}\right)$. The scattering of electrons and blue quarks of the flux tubes in the 2SC cores of compact stars contributes to the transport coefficients of matter and acts as a dissipative force on the flux tubes. The relaxation time for incident light fermion on a flux tube is given by [22]

$$
\tau_{i f}^{-1}=\frac{n_{v}}{p_{F i}} \sin ^{2}\left(\pi \tilde{\beta}_{i}\right)
$$

where $n_{v}$ is the flux density and $p_{F i}$ is the fermion momentum. It is easy to understand the this result. It is of the standard form for classical gases $\tau^{-1}=c n \sigma$, where $c=1$ is the speed of the particles, $n=n_{v}$ is the density of scattering centers, and $\sigma \propto \sin ^{2}(\pi \tilde{\beta}) / p_{F}$ is the cross section for Aharonov-Bohm scattering. One of the blue quarks, the blue down quark, has no A-B interaction with the flux tubes $(\tilde{\beta}=0)$. The other two, the electron and blue up quark, have identical A-B factors although their Fermi momenta are different.

Because the ambient magnetic field in a neutron star is below the lower critical field required to force $X$-flux tubes into $2 \mathrm{SC}$ quark matter, the trapped flux tubes will feel a boundary force pulling them outwards. The outward force per unit length on the flux tube is $f_{b}=r \varepsilon_{X} /\left(R^{2}-r^{2}\right)$ where $\varepsilon_{X}$ is the energy per length of $X$ flux tubes (flux tension), $R$ is the radius of the $2 \mathrm{SC}$ core, $r$ is the radial coordinate in cylindrical coordinates. Other contributions to the magnetic field from outside of the 2SC core have been neglected. Substituting the flux tension we obtain [22]

$$
f_{b} \approx \frac{1}{3 \pi}\left(\frac{r}{R^{2}-r^{2}}\right) \mu_{q}^{2} \ln \kappa_{X}
$$

where $\kappa_{X}$ is the Ginzburg-Landau parameter. This force will be balanced by the drag force ("mutual friction") on the moving flux tube due to its Aharonov-Bohm interaction with the thermal population of gapless quarks and electrons, and also by the MagnusLorentz force. An estimate of the timescale for the expulsion of $X$ flux tubes from a 2SC core on the basis of balance of forces acting on the vortex shows that it is of order $10^{10}$ years [22]. Therefore, it is safe to assume that the magnetic field will be trapped in the 2SC core over evolutionary timescales in the absence of other external forces.

\section{FINAL REMARKS}

There has been substantial progress in understanding the astrophysics of compact objects containing quark matter in their interiors over the past years. However much remains to be done. Further work is needed in developing methods to treat quark matter in non-perturbative regime relevant to compact stars. Finding its ground state in the cold and dense regime and within realistic models is another long-standing challenge. Astrophysics offers several avenues for discerning the state of the matter hidden inside compact stars. Some of them such as measurements of their gross parameters, gravitational radiation and magnetism have been discussed above. Other sensitive tools provided by nature are the thermal cooling and anomalies in rotation of compact star. These issues are discussed elsewhere [30]. 


\section{ACKNOWLEDGMENTS}

I thank M. Alford, N. Ippolito, B. Knippel, M. Ruggieri, D. H. Rischke, and F. Weber for their contributions to the research described in this article and M. Alford for comments on the manuscript. I am grateful to the organizers of QCD@Work Giuseppe Nardulli memorial workshop for their impressive efforts in arranging a successful conference. This work was supported, in part, by the Deutsche Forschungsgemeinshaft and by the European research netwrok "Compstar".

\section{REFERENCES}

1. Pairing in Fermionic Systems, edited by A. Sedrakian, J. W. Clark and M. Alford, (World Scientific, Singapore, 2006).

2. D. Bailin and A. Love, Phys. Rept. 107, 325 (1984).

3. M. G. Alford, A. Schmitt, K. Rajagopal, and T. Schafer, Rev. Mod. Phys. 80, 1455 (2008).

4. R. Casalbuoni and G. Nardulli, Rev. Mod. Phys. 76, 263 (2004).

5. N. Ippolito, M. Ruggieri, D. H. Rischke, A. Sedrakian and F. Weber, Phys. Rev. D 77, 023004 (2008).

6. F. Weber, Pulsars as astrophysical laboratories for nuclear and particle physics, Bristol, U.K.: Institute of Physics, 1999.

7. A. Sedrakian, Prog. Part. Nucl. Phys. 58, 168 (2007).

8. M. Alford and S. Reddy, Phys. Rev. D 67, 074024 (2003).

9. B. K. Agrawal, Phys. Rev. D 81, 023009 (2010).

10. A. G. Lyne et al., Science, 303, 1153 (2004).

11. M. Burgay et al., Nature 426, 531 (2003).

12. J. M. Lattimer and B. F. Schutz, Astrophys. J. 629, 979 (2005).

13. R. X. Xu, Astrophys. J. 596, L59 (2003).

14. B. J. Owen, Phys. Rev. Lett. 95, 211101 (2005).

15. B. J. Owen, Class. Quant. Grav. 26, 204014 (2009).

16. M. Mannarelli, K. Rajagopal and R. Sharma, Prog. Theor. Phys. Suppl. 174, 39 (2008).

17. L.-M. Lin, Phys. Rev. D 76, 081502(R) (2007).

18. B. Haskell, N. Andersson, D. I. Jones, and L. Samuelsson, Phys. Rev. Lett. 99, 231101 (2007).

19. B. Knippel and A. Sedrakian, Phys. Rev. D 79, 083007 (2009).

20. B. Abbott, et al. (LIGO Scientific Collaboration) Astrophys. J. Letters 683, 45 (2008).

21. C. J. Horowitz, Phys. Rev. D 81, 103001 (2010).

22. M. G. Alford and A. Sedrakian, J. Phys. G 37, 075202 (2010).

23. M. G. Alford, J. Berges and K. Rajagopal, Nucl. Phys. B 571, 269 (2000).

24. K. Iida and G. Baym, Phys. Rev. D 66, 014015 (2002).

25. D. M. Sedrakian, D. Blaschke, K. M. Shahabasian and D. N. Voskresensky, Phys. Part. Nucl. 33, S100 (2002); Astrofiz. 44, 443 (2001) [Astrophysics 44, 359 (2001)].

26. T. Vachaspati, Phys. Rev. Lett. 68, 1977 (1992).

27. M. James, L. Perivolaropoulos, and T. Vachaspati, Nucl. Phys. B395, 534 (1993).

28. T. Vachaspati and R. Watkins, Phys. Lett. B318, 163 (1993).

29. M. G. Alford and F. Wilczek, Phys. Rev. Lett. 62, 1071 (1989).

30. A. Sedrakian, Acta Phys. Polon. B 3, 669 (2010). 